Engineering and Computational Mechanics Volume 166 Issue EM2

Stress analysis without meshing: isogeometric boundary-element method Lian, Simpson and Bordas
Proceedings of the Institution of Civil Engineers

Engineering and Computational Mechanics 166 June 2013 Issue EM2 Pages 88-99 http://dx.doi.org/10.1680/eacm.11.00024

Paper 1100024

Received $17 / 11 / 2011$

Received 17/11/2011
Published online 03/04/2013

Keywords: computational mechanics/design method \& aids/stress analysis

ICE Publishing: All rights reserved i

ice

Institution of Civil Engineers

\title{
Stress analysis without meshing: isogeometric boundary-element method
}

1 Haojie Lian BEng, MSc

PhD Student, School of Engineering, Cardiff University, Cardiff, UK

2. Robert N. Simpson MEng, PhD

Lecturer, School of Engineering, Cardiff University, Cardiff, UK
3 Stéphane P. A. Bordas MSc, PhD

Professor, School of Engineering, Cardiff University, Cardiff, UK

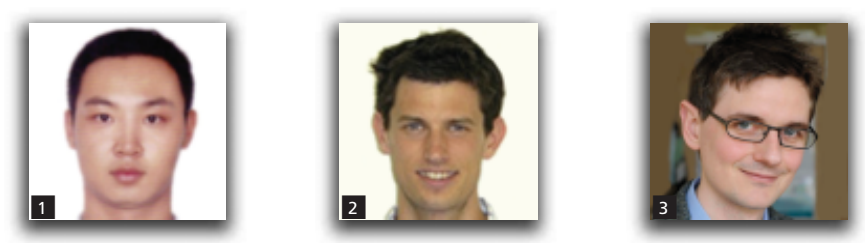

The focus of this paper is the description and numerical validation of a computational method where stress analysis can be performed directly from computer-aided design data without mesh generation. The clear benefit of the approach is that no mesh needs to be generated prior to running the analysis. This is achieved by utilising the isogeometric concept whereby computer-aided design data are used to construct not only the geometry discretisation but also the displacement and traction approximations. In this manner, significant savings can be made in the engineering design and analysis process. This paper also demonstrates that, compared with a standard boundary-element method implementation using quadratic Lagrangian shape functions, superior accuracy is achieved using the present approach for the same number of degrees of freedom. It further illustrates practical applications of the method, comparing against results obtained with a standard boundary-element method and finite-element method for verification. In addition, a propeller is analysed as a sample to show the ability of the present method to handle complex three-dimensional geometries.

\section{Notation}

a basis function index

$\mathbf{B}_{a} \quad$ control point coordinate

$C_{i j} \quad$ jump term

$d_{j}^{a} \quad$ displacement coefficient component

$N_{a, p} \quad$ B-spline basis function

$n \quad$ basis function number

$p \quad$ basis function order

$q_{j}^{a} \quad$ traction coefficient component

$R_{a, p} \quad$ non-uniform rational B-splines (NURBS) basis function

$T_{i j} \quad$ traction fundamental solution

$t_{i} \quad$ traction component

$U_{i j} \quad$ displacement fundamental solution

$u_{i} \quad$ displacement component

$w_{a} \quad$ NURBS weight

$\mathbf{x}^{\prime}$

\section{Introduction}

The finite-element method (FEM) (Strang and Fix, 1973; Zienkiewicz, 1971) and boundary-element method (BEM) (Banerjee and Butterfield, 1981) are two numerical techniques that have seen extensive development for engineering analysis. The FEM is applicable to a wide variety of engineering problems and has enjoyed much commercial success since its inception. The BEM possesses certain advantages over the FEM due to the requirement for only a boundary discretisation (in contrast to a domain discretisation for the FEM), essentially reducing the dimensionality of the problem. This is at the cost of a full matrix 'inversion' and technicalities related to numerical integration. In conventional implementations, both methods use polynomial functions to create a discretisation of the geometry and unknown fields (e.g. displacement), requiring a pre-processing procedure known as 'meshing' to be carried out. To create an appropriate 'analysis-ready' mesh is costly and time consuming, particularly in the case of complicated three-dimensional (3D) domains where large numerical errors can result if an appropriate mesh is not constructed.

To suppress the need to generate analysis-ready meshes, the 
concept of isogeometric analysis (IGA) (Hughes et al., 2005) was introduced to FEM (IGAFEM). The key ideas behind such an approach are as follows.

- The same basis functions as used by computer-aided design (CAD) (e.g. non-uniform rational B-splines (NURBS) (Piegl and Tiller, 1997), T-splines (Sederberg et al., 2003) etc.) are used to approximate not only the geometry of the domain, but also the unknown fields.

- The unknown fields then become associated with control points (used to define the CAD geometry) rather than nodal points.

- The geometry of the problem is defined exactly at all stages of analysis.

Since this seminal development, IGAFEM has been applied successfully in many other areas including structural analysis (Cottrell et al., 2006), shape optimisation (Wall et al., 2008), shell analysis (Benson et al., 2010), contact problems (Temizer et al., 2010) and electromagnetics (Buffa et al., 2010).

However, in IGAFEM, a mismatch still remains between the information provided by $\mathrm{CAD}$ and the discretisation required for numerical analysis. The FEM requires a domain representation of the geometry while CAD provides only a surface representation requiring certain pre-processing steps to be carried out. In the case of the BEM where only a surface representation is required for analysis, it is found that the isogeometric concept is a particularly nice fit since both deal with quantities defined entirely on the boundary. The first isogeometric BEM (IGABEM) (Simpson et al., 2012) for two-dimensional (2D) elastostatic analysis was proposed in 2011, and more accurate results per degree of freedom were achieved compared with a conventional BEM using quadratic Lagrangian shape functions. Compared with IGAFEM, IGABEM possesses the particular advantage that CAD data can be used directly for analysis without the need to generate a discretisation of the domain.

In this paper, the authors utilise the flexible properties of IGABEM to analyse civil engineering structures where the benefits over conventional BEM and FEM procedures are demonstrated. The paper is organised as follows. Section 2 presents some basic knowledge of non-uniform rational B-splines, the parametric functions predominant in CAD. The IGABEM is outlined in Section 3, and three numerical examples to demonstrate the efficiency and accuracy of the method are given in Section 4. The paper concludes with Section 5.

\section{B-spline curves and non-uniform rational B-splines}

Since isogeometric methods rely on the use of basis functions generated by $\mathrm{CAD}$, some discussion of such functions is in order here. The predominant functions are non-uniform rational Bsplines (NURBS), but the algorithms used for their evaluation are extended from those used for B-splines. Both B-splines and
NURBS are thus introduced, highlighting certain features useful for analysis.

\section{$2.1 \quad$ B-spline curves}

B-splines can be considered a subset of NURBS. They are affine mappings from the parametric space to the physical space. The expression of a B-spline curve can therefore be written as

1. $\mathbf{C}(\xi)=\sum_{a=1}^{n} N_{a, p}(\xi) \mathbf{B}_{a}$

where $\xi$ denotes the parametric space coordinate, $\mathbf{B}_{a}$ the control point coordinates, $n$ the number of basis functions, $\mathbf{C}$ the global coordinates interpolated by the curve and $N_{a, p}$ the B-spline basis functions where $a$ denotes the index of the basis function and $p$ the order of the basis function (see Figures 1 and 2).

In light of Equation 1, it can be seen that a B-spline curve is determined by three things.

- Control points. These do not necessarily lie on the boundary of the domain. The piecewise linear interpolation of the control points generates the control polygon. The control polygon is useful for interactive design because it provides intuitive geometrical information.

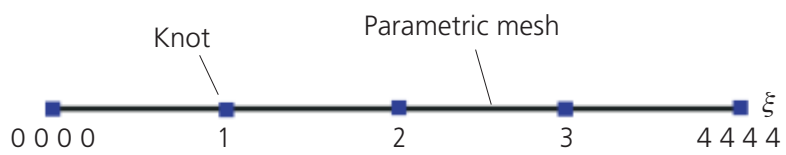

Figure 1. Parametric representation of B-spline

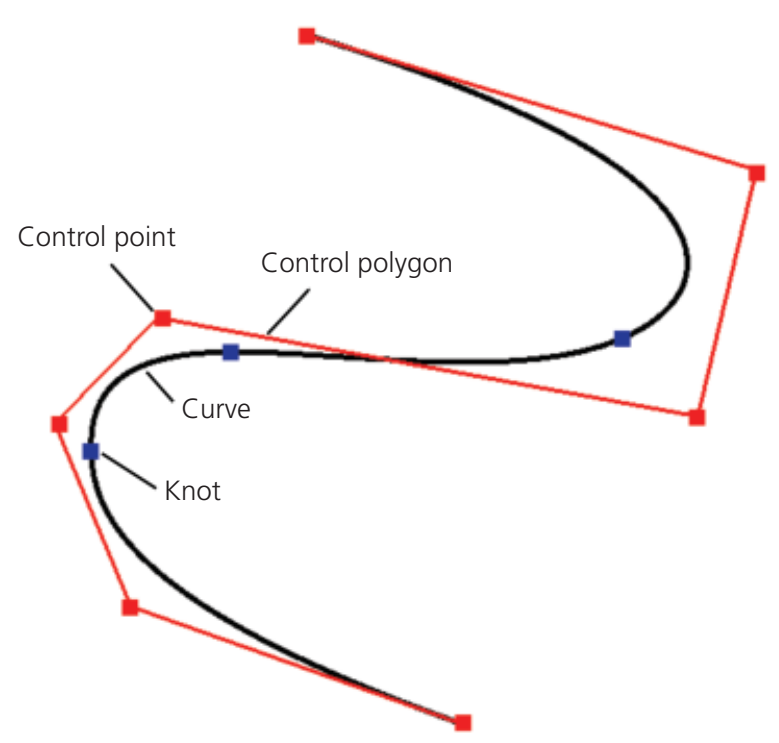

Figure 2. Physical representation of B-spline 
- Basis functions. Every basis function is associated with a control point. The basis function plays a key role in IGA, which will be detailed in the next section.

- Parametric space. The parametric space is always structured. It is a straight line, rectangle or cuboid in one-dimensional, $2 \mathrm{D}$ or $3 \mathrm{D}$ spaces respectively. In some cases, the physical space is a mapping from more than one parametric space. In this case, the problem is referred to as a multiple-patch problem where each parametric space is called a patch.

\subsection{B-spline basis functions}

Before the introduction of B-spline basis functions, it is necessary to start with the concept of a knot vector, which has a direct influence on the resulting basis functions.

A knot vector is defined as a set of non-decreasing real numbers in the parametric space

$$
\left\{\xi_{1}, \xi_{2}, \ldots, \xi_{n+p+1}\right\} \quad \xi_{i} \in \mathbb{R}
$$

where $i$ denotes the knot index, $p$ is the curve order and $n$ is the number of basis functions or control points. Each real number $\xi_{i}$ is called a knot. The number of knots is given by $m=n+p+1$. The half-open interval $\left[\xi_{i}, \xi_{i+1}\right)$ is called a knot span.

Within the knot vector, knots can be repeated where, for example, $\{0,0,0,1,1,2,2,3,3,3\}$ is a valid knot vector. Knots with different values can be viewed as different break points that divide the parametric space into different elements. Hence, the physical interpretation of the knots can be explained as the parametric coordinates of the element edges, while the 'knot span' between two knots with different values can be viewed as the definition of elements in the parametric space. The insertion of a new knot will split an element, much like $h$-refinement in the FEM. However, the repetition of existing knots will not increase the number of elements, but can be used to decrease the order of the basis functions. For example, the knot vector $\{0,0,0,1,1,2,2,3,3,3\}$ has ten knot values and nine knot spans, $[0,0),[0,0),[0,1),[1,1),[1,2),[2,2),[2,3),[3,3)$ and $[3,3)$, but only three elements, $[0,1],[1,2]$ and $[2,3]$.

The knot vector is open if its first and last knot values are repeated $p+1$ times, such as $\{0,0,0,1,2,3,4,4,4\}$. The open knot vector is the standard in $\mathrm{CAD}$, so all the examples in this paper use open knot vectors. The knot vector values can be normalised without affecting the resulting B-spline. Therefore, $\{0,0,0,1,2,3,4,4,4\}$ is equivalent to $\{0,0,0,1 / 4,2 / 4,3 / 4$, $1,1,1\}$. It is called a uniform knot vector if the knots are uniformly spaced, for example $\{0,0,0,1,2,3,4,5,5,5\}$.

It is necessary to differentiate control points and knots in IGA with nodes in the standard FEM or BEM. In the standard FEM and BEM, nodes are placed on the domain or the boundary to discretise the geometry and the unknown fields. In IGA, the equivalent of a node is a control point, which may lie outside the domain. The knot values are used to divide the space into elements.

With the concept of a knot vector, we can now define B-spline basis functions. There exist numerous definitions of B-spline basis functions but, for convenience in implementation, the Coxde Boor recursion formula (Cox, 1971; de Boor, 1972) is used here

2. $\quad N_{a, 0}(\xi)= \begin{cases}1, & \text { if } \xi_{a} \leqslant \xi<\xi_{a+1} \\ 0, & \text { otherwise }\end{cases}$

3.

$$
\begin{aligned}
N_{a, p}(\xi)= & \frac{\xi-\xi_{a}}{\xi_{a+p}-\xi_{a}} N_{a, p-1}(\xi) \\
& +\frac{\xi_{a+p+1}-\xi}{\xi_{a+p+1}-\xi_{a+1}} N_{a+1, p-1}(\xi)
\end{aligned}
$$

In essence, a B-spline basis function is a piecewise polynomial function. The functions are $C^{\infty}$ within elements and $C^{p-m}$ on element boundaries, where $m$ is the number of knot repetitions.

From Figure 3, the following properties of B-spline basis functions can be observed.

- Local support - the B-spline basis function $N_{a, p}$ is always non-negative in knot spans of $\left[\xi_{a}, \xi_{a+p+1}\right.$ ) This has an important significance for interactive design: the change of one control point only affects the local part of the curve, giving great convenience for curve modification.

- Non-interpolatory - the B-spline basis functions do not interpolate the control points except at the start point, end point and any point whose knot value is repeated $p$ times.

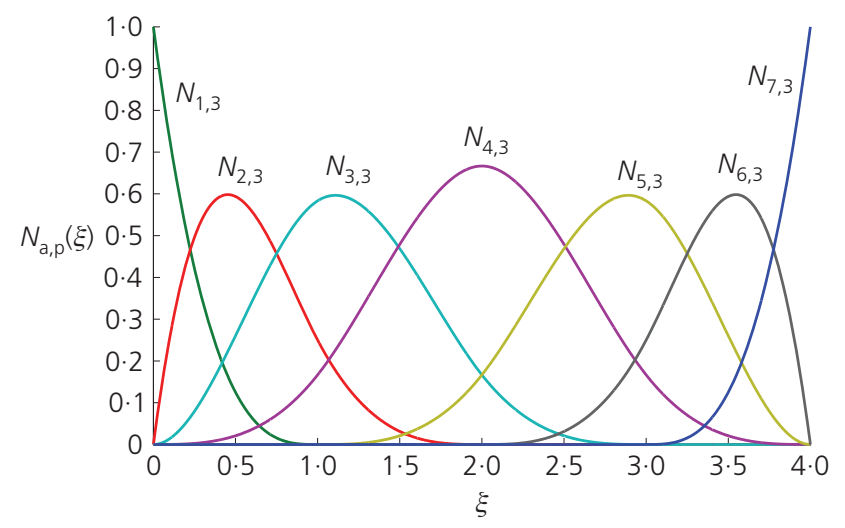

Figure 3. B-spline basis functions with knot vector $\{0,0,0,0,1,2,3,4,4,4,4\}$ 
The continuity and differentiability of a B-spline curve is inherited directly from its basis functions where it is found that the continuity of a B-spline curve is at least $C^{p-m}$.

\subsection{NURBS}

NURBS are important parametric curves in CAD and are seen as the industry standard with implementation in several commercial software packages. In addition, all numerical examples in this paper are represented by NURBS. NURBS are developed from B-spline curves but can offer significant advantages due to their ability to represent a wide variety of geometric entities. The expression defining NURBS interpolation is very similar to that of B-splines

4.

$$
\mathbf{C}(\xi)=\sum_{a=1}^{n} R_{a, p}(\xi) \mathbf{B}_{a}
$$

in which $\mathbf{B}_{a}$ is the set of control point coordinates and $R_{a, p}$ are NURBS basis functions, defined as

5.

$$
R_{a, p}(\xi)=\frac{N_{a, p}(\xi) w_{a}}{W(\xi)}=\frac{N_{a, p}(\xi) w_{a}}{\sum_{\hat{a}=1}^{n} N_{\hat{a}, p} w_{\hat{a}}}
$$

where $N_{a, p}$ is the standard B-spline basis function, $W(\xi)$ is the weighting function, and $w_{a}$ is the weight that is associated with $N_{a, p}$ and influences the distance between the curve and control points, with higher values drawing the curve closer to that point (see Figures 4 and 5). When all of the weights are equal to 1, the NURBS reduces to a B-spline curve. The NURBS basis function is a piecewise rational function.

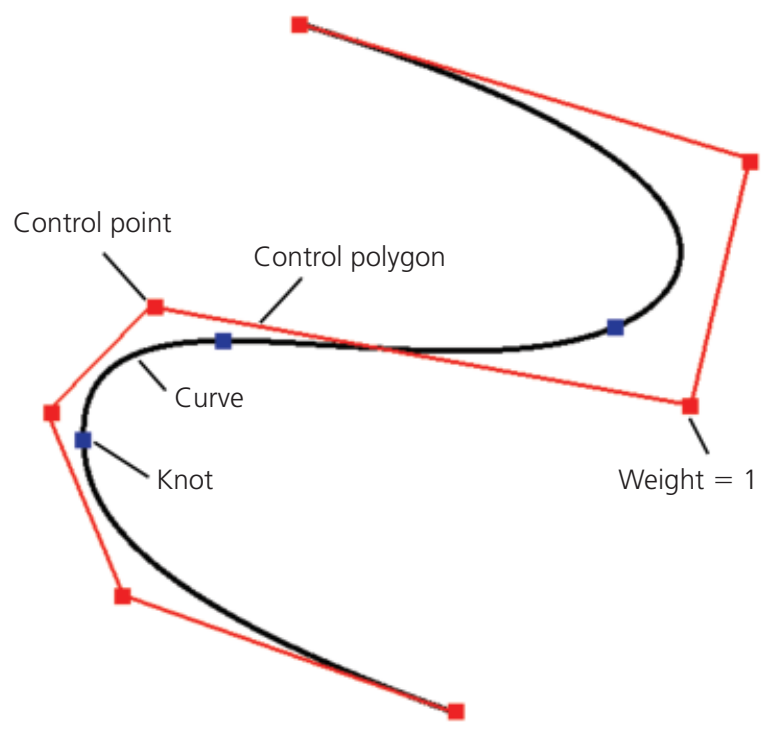

Figure 4. NURBS with weights $\{1,1,1,1,1,1,1\}$

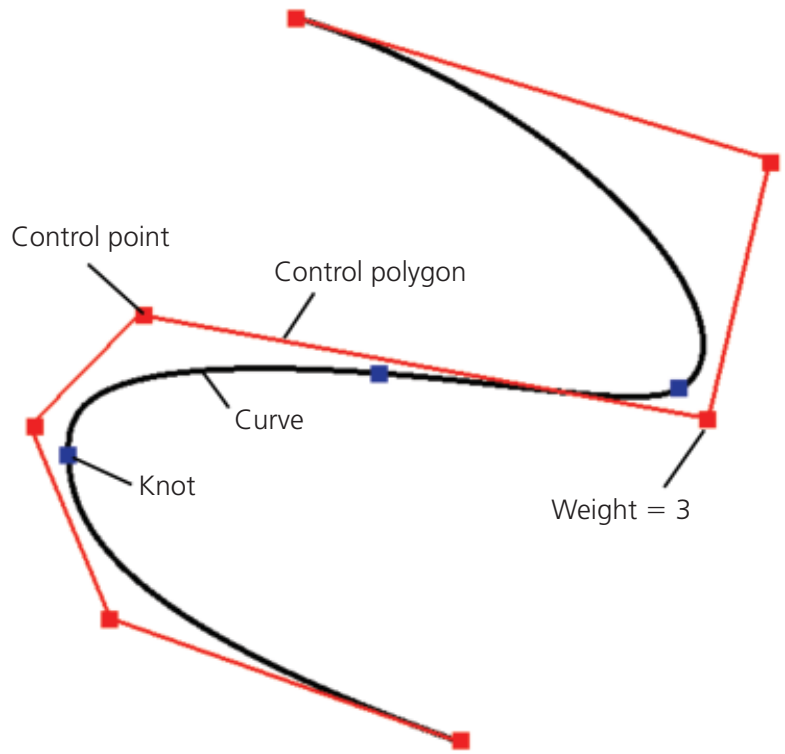

Figure 5. NURBS with weights $\{1,1,1,1,3,1,1\}$

\subsection{Multiple-patch problem}

For some complex geometries, especially for multiply connected domains, the geometry is obtained by the mapping from multiple parametric spaces. In this case, each parametric space is called a patch. IGABEM possesses advantages over IGAFEM for problems with multiple patches. In IGAFEM, the geometry is the domain representation, and thus the plate in Figure 6 is a plane divided into four patches. To guarantee geometric continuity we must join patches along each of the patch boundaries. Using current geometrical algorithms, only $C^{0}$ continuity along each of the patch boundaries can be guaranteed. For the boundary representation, the geometry of the same example is determined



Figure 6. Multiple-patch problem of plate with four holes 
by two curves - an outer boundary and inner boundary - which are two geometrically independent patches and therefore do not need to be connected.

\section{IGABEM formulation}

The idea of IGA relies on the fact that the geometric representation in CAD can also be used to approximate the unknown fields in numerical simulation. The only difference is that, in computational geometry, the nodal parameters are the coordinates of the control points in the physical space, but in analysis they are associated with unknown field variables. A natural idea is to use the same control points and the same basis functions to discretise the unknown fields.

Let us consider 2D linear elastostatic problems as an example to derive the equations of the isogeometric BEM. The displacement boundary integral equation is

$$
\begin{aligned}
& C_{i j}\left(\mathbf{x}^{\prime}\right) u_{j}\left(\mathbf{x}^{\prime}\right)+f_{\Gamma} T_{i j}\left(\mathbf{x}^{\prime}, \mathbf{x}\right) u_{j}(\mathbf{x}) \mathrm{d} \mathbf{x} \\
& \text { 6. } \quad=\int_{\Gamma} U_{i j}\left(\mathbf{x}^{\prime}, \mathbf{x}\right) t_{j}(\mathbf{x}) \mathrm{d} \mathbf{x}
\end{aligned}
$$

where $\mathbf{x}^{\prime}$ is the source point, $\mathbf{x}$ is the field point and $\Gamma$ is the boundary. $U_{i j}$ and $T_{i j}$ are the fundamental solutions, which depend on the material properties and the distance between $\mathbf{x}^{\prime}$ and $\mathbf{x} . u_{j}$, and $t_{j}$ are the components of the displacement and traction around the boundary, respectively. The physical significance of the fundamental solution is the influence of a concentrated point force at a given source point on the field point. $C_{i j}$ is the jump term, which only depends on the geometry of the boundary at the source point, and $f$ represents integration in the Cauchy principal value limiting sense.

Discretising the displacement and the traction fields with NURBS basis functions yields

$$
u_{j}(\xi)=\sum_{a=1}^{n} R_{a, p}(\xi) d_{j}^{a}=\sum_{a=1}^{n} R_{a}(\xi) d_{j}^{a}
$$

8.

$$
t_{j}(\xi)=\sum_{a=1}^{n} R_{a, p}(\xi) q_{j}^{a}=\sum_{a=1}^{n} R_{a}(\xi) q_{j}^{a}
$$

where subscript $p$ in the basis function has been omitted for simplicity and $d_{j}^{a}$ and $q_{j}^{a}$ denote the nodal parameters related to displacement and traction, respectively. Every nodal parameter corresponds to a control point. Substituting Equations 7 and 8 into the displacement boundary integral equation (Equation 6) yields the system of equations

$$
\begin{aligned}
& \sum_{a=1}^{n}\left[C_{i j}\left(\mathbf{x}^{\prime}\right) R_{a}\left(\xi^{\prime}\right)\right] d_{j}^{a} \\
& +\sum_{a=1}^{n}\left[f_{\gamma} T_{i j}\left(\mathbf{x}^{\prime}, \mathbf{x}(\xi)\right) R_{a}(\xi) J(\xi) \mathrm{d} \xi\right] d_{j}^{a} \\
& =\sum_{a=1}^{n}\left[\int_{\gamma} U_{i j}\left(\mathbf{x}^{\prime}, \mathbf{x}(\xi)\right) R_{a}(\xi) J(\xi) \mathrm{d} \xi\right] q_{j}^{a}
\end{aligned}
$$

where $\xi^{\prime}$ denotes the location of the source point in parametric space, $\gamma$ is the parametric representation of $\Gamma$ and $J$ is the determinant of the Jacobian matrix. Because the basis function $R_{a}$ is locally supported, the integration is performed in a piecewise manner. In addition, the domain of integration will be mapped into the domain $[-1,1]$ to allow Gauss-Legendre quadrature to be used.

Equation 9 can be written in matrix notation as

10. $[\mathbf{H}]\{\mathbf{u}\}=[\mathbf{G}]\{\mathbf{t}\}$

$$
H_{i j}^{a}=f_{\gamma}\left[T_{i j}\left(\mathbf{x}^{\prime}, \mathbf{x}(\xi)\right) R_{a}(\xi) J(\xi)\right] \mathrm{d} \xi
$$

11.

$$
+C_{i j}\left(\mathbf{x}^{\prime}\right) R_{a}\left(\xi^{\prime}\right)
$$

12.

$$
G_{i j}^{a}=\int_{\gamma}\left[U_{i j}\left(\mathbf{x}^{\prime}, \mathbf{x}(\xi)\right) R_{a}(\xi) J(\xi)\right] \mathrm{d} \xi
$$

$[\mathbf{H}]$ is a coefficient matrix calculated from the jump terms and integral of $T_{i j}$ for every collocation point, $\mathbf{u}$ is the column vector containing all the displacement nodal unknowns $d_{j}^{a}$. [G] is a coefficient matrix containing the integral of $U_{i j}$ and $\mathbf{t}$ is the column vector containing all the traction nodal unknowns $q_{j}^{a}$.

The strongly singular integration and weakly singular integration need to be evaluated for $[\mathbf{H}]$ and $[\mathbf{G}]$, respectively. Owing to the local support properties of B-spline basis functions, the singularity integration is only performed for the coefficients associated with the nodal unknowns whose basis function support contains the element that the collocation point resides in.

The following expressions illustrate the matrix entries

13

$$
\bar{H}_{i j}^{a c}=\int_{\gamma} T_{i j}\left(\mathbf{x}^{c}, \mathbf{x}(\xi)\right) R_{a}(\xi) J(\xi) \mathrm{d} \xi
$$


14. $\tilde{H}_{i j}^{a c}=C_{i j}\left(\mathbf{x}^{c}\right) R_{a}\left(\xi^{\prime}\right)$

where $c$ is a collocation point index. Consider an arbitrary closed curve with open knot vector $\{0,0,0,1,2,3,4,4,5,5,5\}$. The parametric coordinates of control points can be chosen as $\{0,0 \cdot 5,1 \cdot 5,2 \cdot 5,3 \cdot 5,4,4 \cdot 5\}$. Therefore, the $[\mathbf{H}]$ matrix entries are

15. $\left[\begin{array}{ccccccc}\bar{H}_{i j}^{11}+\bar{H}_{i j}^{81}+\tilde{H}_{i j}^{11} & \bar{H}_{i j}^{21} & \bar{H}_{i j}^{31} & \bar{H}_{i j}^{41} & \bar{H}_{i j}^{51} & \bar{H}_{i j}^{61} & \bar{H}_{i j}^{71} \\ \bar{H}_{i j}^{12}+\bar{H}_{i j}^{82}+\tilde{H}_{i j}^{12} & \bar{H}_{i j}^{22}+\tilde{H}_{i j}^{22} & \bar{H}_{i j}^{32}+\tilde{H}_{i j}^{32} & \bar{H}_{i j}^{42} & \bar{H}_{i j}^{52} & \bar{H}_{i j}^{62} & \bar{H}_{i j}^{72} \\ \bar{H}_{i j}^{13}+\bar{H}_{i j}^{83} & \bar{H}_{i j}^{23}+\tilde{H}_{i j}^{23} & \bar{H}_{i j}^{33}+\tilde{H}_{i j}^{33} & \bar{H}_{i j}^{43}+\tilde{H}_{i j}^{43} & \bar{H}_{i j}^{53} & \bar{H}_{i j}^{63} & \bar{H}_{i j}^{73} \\ \bar{H}_{i j}^{14}+\bar{H}_{i j}^{84} & \bar{H}_{i j}^{24} & \bar{H}_{i j}^{34}+\tilde{H}_{i j}^{34} & \bar{H}_{i j}^{44}+\tilde{H}_{i j}^{44} & \bar{H}_{i j}^{54}+\tilde{H}_{i j}^{54} & \bar{H}_{i j}^{64} & \bar{H}_{i j}^{74} \\ \bar{H}_{i j}^{15}+\bar{H}_{i j}^{85} & \bar{H}_{i j}^{25} & \bar{H}_{i j}^{35} & \bar{H}_{i j}^{45}+\tilde{H}_{i j}^{45} & \bar{H}_{i j}^{55}+\tilde{H}_{i j}^{55} & \bar{H}_{i j}^{65}+\tilde{H}_{i j}^{65} & \bar{H}_{i j}^{75} \\ \bar{H}_{i j}^{16}+\bar{H}_{i j}^{86} & \bar{H}_{i j}^{26} & \bar{H}_{i j}^{36} & \bar{H}_{i j}^{46} & \bar{H}_{i j}^{56} & \bar{H}_{i j}^{66}+\tilde{H}_{i j}^{66} & \bar{H}_{i j}^{76} \\ \bar{H}_{i j}^{17}+\tilde{H}_{i j}^{87} & \bar{H}_{i j}^{27} & \bar{H}_{i j}^{37} & \bar{H}_{i j}^{47} & \bar{H}_{i j}^{57} & \bar{H}_{i j}^{67}+\tilde{H}_{i j}^{67} & \bar{H}_{i j}^{77}+\tilde{H}_{i j}^{77}\end{array}\right]$

The boundary conditions are applied by placing all unknowns on the left-hand side and all known values on the right-hand side. To visualise this, it is convenient to write Equation 10 as

16. $\left[\begin{array}{ll}\mathbf{H}_{1} & \mathbf{H}_{2}\end{array}\right]\left\{\begin{array}{l}\mathbf{u}_{1} \\ \mathbf{u}_{2}\end{array}\right\}=\left[\begin{array}{ll}\mathbf{G}_{1} & \mathbf{G}_{2}\end{array}\right]\left\{\begin{array}{l}\mathbf{t}_{1} \\ \mathbf{t}_{2}\end{array}\right\}$

where the indices 1 and 2 denote the values corresponding to the Neumann and Dirichlet boundaries, respectively. All unknowns are moved to the left-hand side and all known values are moved to the right-hand side, giving

17. $\left[-\mathbf{G}_{1} \mathbf{H}_{2}\right]\left\{\begin{array}{c}\mathbf{t}_{1} \\ \mathbf{u}_{2}\end{array}\right\}=\left[-\mathbf{H}_{1} \mathbf{G}_{2}\right]\left\{\begin{array}{c}\mathbf{u}_{1} \\ \mathbf{t}_{2}\end{array}\right\}$

Performing matrix-vector multiplication on the right-hand side, the terms in this equation are denoted as

18. $[\mathbf{A}]=\left[\begin{array}{ll}-\mathbf{G}_{1} & \mathbf{H}_{2}\end{array}\right]$

19. $\{\mathbf{x}\}=\left\{\begin{array}{l}\mathbf{t}_{1} \\ \mathbf{u}_{2}\end{array}\right\}$

20.

$\{\mathbf{b}\}=\left[\begin{array}{ll}-\mathbf{H}_{1} & \mathbf{G}_{2}\end{array}\right]\left\{\begin{array}{l}\mathbf{u}_{1} \\ \mathbf{t}_{2}\end{array}\right\}$ giving the final system of equations

21. $[\mathbf{A}]\{\mathbf{x}\}=\{\mathbf{b}\}$

Figure 7 is the flowchart of the IGABEM implementation for a single-patch problem. The shaded blocks indicate different parts from standard BEM. The multiple-patch implementation is similar, except that an additional loop is added over all the patches before the loop over the collocation points. The code structure of IGABEM preserves the basic framework of the standard BEM, so it can be incorporated easily into any BEM code.

\section{Numerical examples}

\subsection{Pressure vessel}

The first example is a pressure vessel, which is a 2D plain strain problem. Due to symmetry of the problem, only a quarter of the pressure vessel is studied, as illustrated in Figure 8 where the geometry, material properties and boundary conditions are also defined. A second-order approximation $(p=2)$ is chosen. In this case, the minimum number of control points is shown in Figure 9. Appendix 1 provides the appropriate coordinates and weights.

The knot vector is defined as

$\{0,0,0,1,1,2,2,3,3,4,4,5,5,6,6,7,7,8,8$, $9,9,10,10,11,11,11\}$

The weights are defined as 


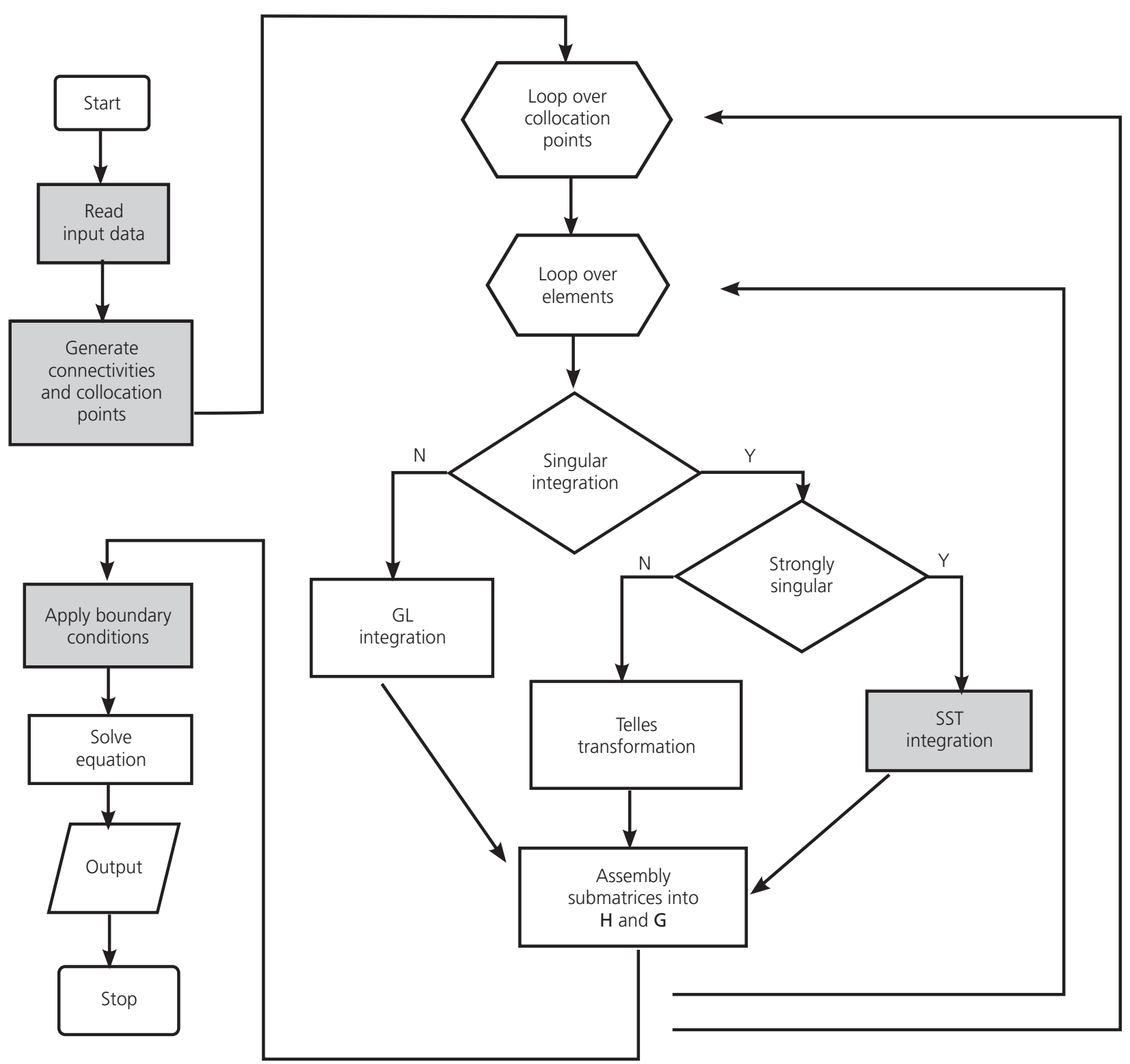

Figure 7. IGABEM flowchart (GL, Gauss-Legendre; SST,

singularity subtraction technique)

$\left\{1,1,1, \frac{2^{1 / 2}}{2}, 1,1,1,1,1,1,1,1,1,1,1,1,1\right.$,

$1,1,1,1,1,1\}$

There is no analytical solution for this problem, but we compare the $L_{2}$ norm of IGABEM

$$
\|\mathbf{u}\|_{L_{2}}=\left(\int_{\Gamma} \sum_{i=1}^{n}\left(u_{i}\right)^{2}\right)^{1 / 2} \mathrm{~d} \Gamma
$$

and that of the BEM with quadratic Lagrangian shape functions, both of which converge to the same solution as shown in Figure 10. Hence, we take this limit as the reference solution to calculate the $L_{2}$ relative error. Figure 11 shows that IGABEM not only achieves more accurate results than the conventional BEM, but also superior convergence. This is an important result, since this shows that, for an equivalent number of degrees of freedom (DOF), IGABEM is more accurate than the conventional quadratic BEM. In addition, the deformed profile of IGABEM compares very favourably with the result obtained with the FEM implementation, as shown in Figure 12. In the FEM implementation, linear triangular elements were used. 
Stress analysis without meshing: isogeometric boundary-element method

Lian, Simpson and Bordas

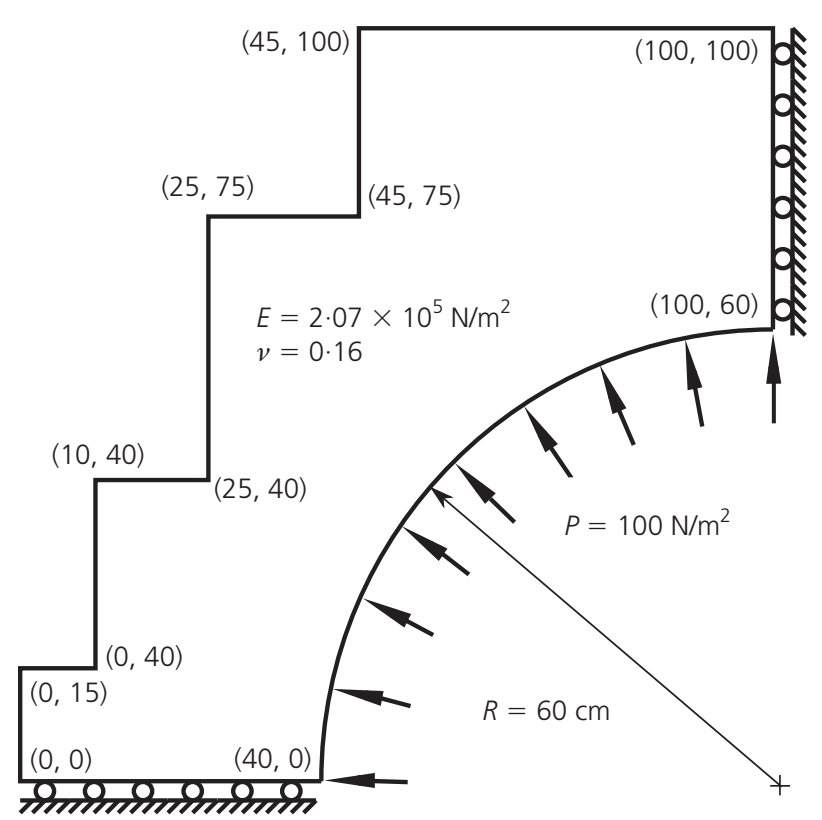

Figure 8. Pressure vessel problem definition

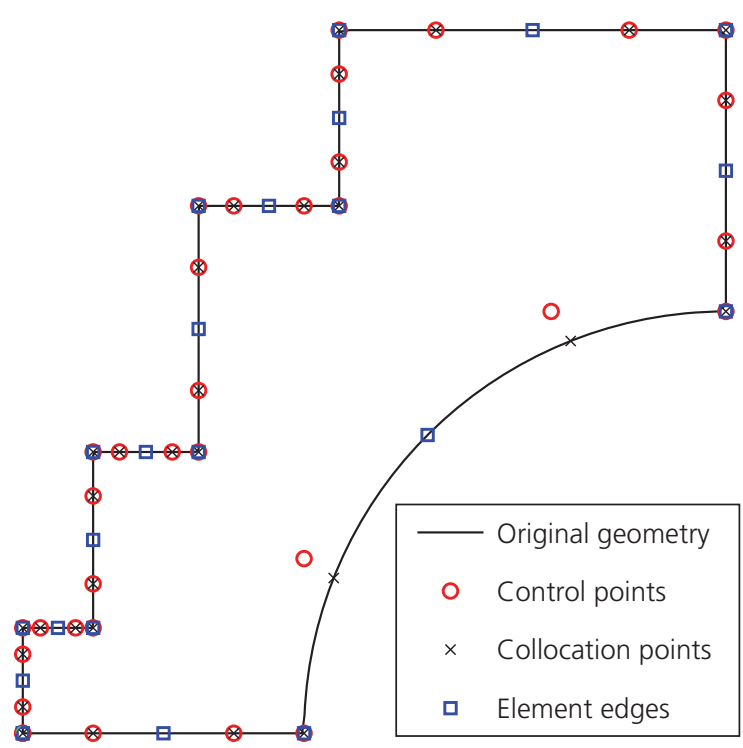

Figure 9. NURBS curve, control points, collocation points and element edges for pressure vessel problem

\subsection{Dam}

The geometry of a dam modelled under plane strain is illustrated in Figure 13 where a hydrostatic loading is present and body forces act throughout the structure. The elastic modulus is $E=1.31 \times 10^{11} \mathrm{~N} / \mathrm{m}^{2}$ with Poisson's ratio $v=0.25$. The hydrostatic water pressure is given by a normal traction $t_{\mathrm{n}}=$ $-[9.81 \times 1000 \times(3.25-y)] \mathrm{N} / \mathrm{m}^{2}$ and tangential traction $t_{\mathrm{t}}=0$. Using a density of $\rho=2300 \mathrm{~kg} / \mathrm{m}^{3}$ and gravitational accelera-

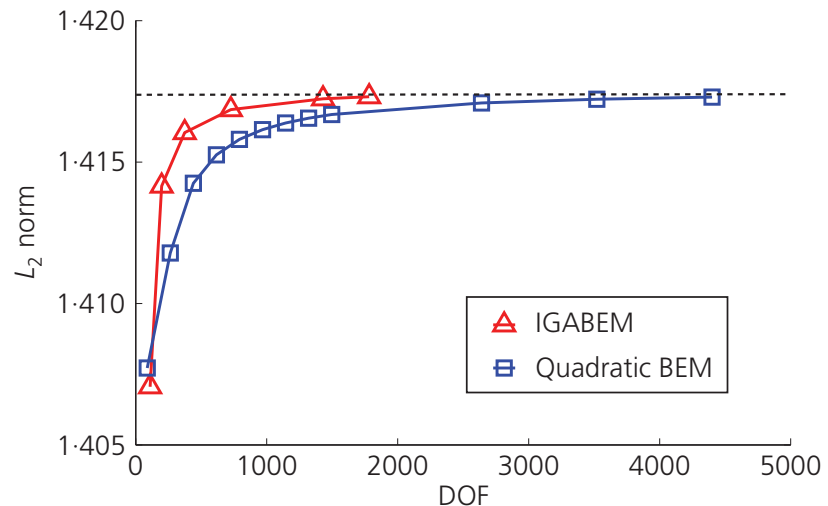

Figure 10. The $L_{2}$ norm of quadratic BEM and IGABEM for pressure vessel problem

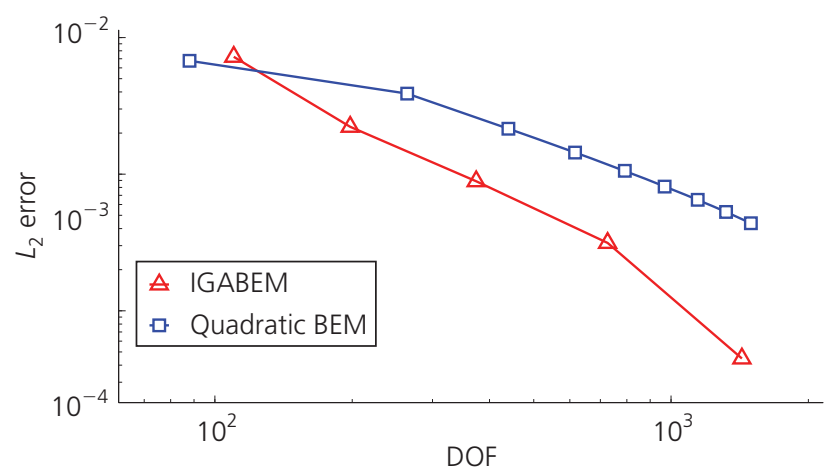

Figure 11. The $L_{2}$ relative error for quadratic BEM and IGABEM for pressure vessel problem

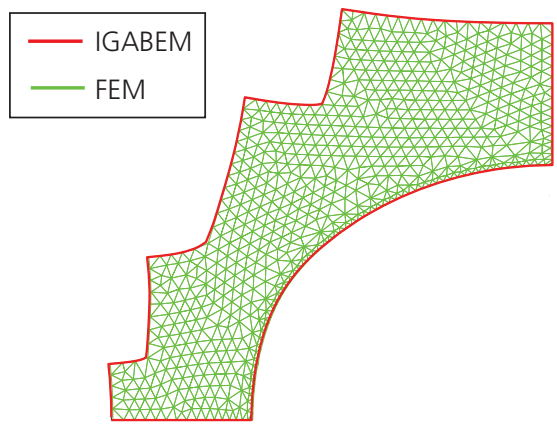

Figure 12. IGABEM and FEM pressure vessel problem: deformed shape

tion of $9.81 \mathrm{~m} / \mathrm{s}^{2}$, the body forces throughout are given by $b_{x}=0$ and $b_{y}=-2300 \times 9.81 \mathrm{~N} / \mathrm{m}^{3}$.

The dam example demonstrates a multiple-patch problem in IGABEM where the boundary of the geometry consists of two curves - an outer boundary and an inner boundary - which form two parametric spaces. In this example, the two curves have the 


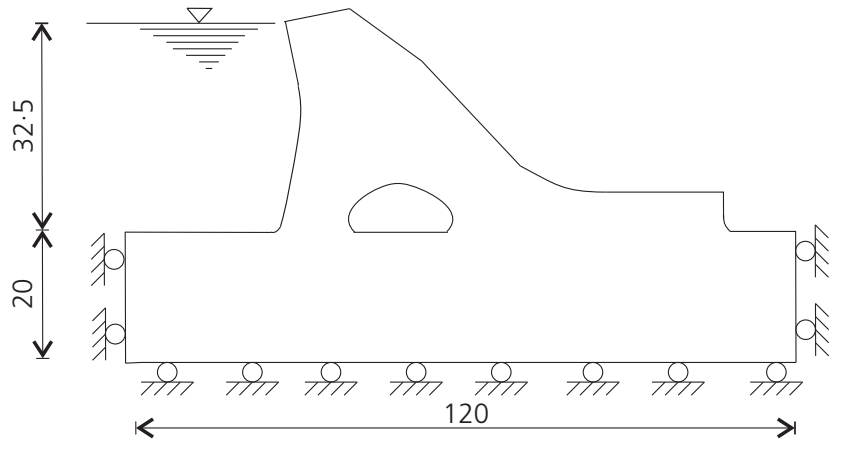

Figure 13. Dam problem definition (dimensions in metres)

same order $(p=2)$ and in the case of two elements per line, the control point coordinates and weights are in Appendix 2 and Appendix 3, respectively.

For the outer boundary, the knot vector is given by

$\{0,0,0,1,1,2,2,3,3,4,4,5,5,6,6,7,7$,

$8,8,9,9,10,10,11,11,12,12,13,13$,

$14,14,15,15,16,16,16\}$

For the inner boundary, the knot vector is given by

$\{0,0,0,1,2,3,4,5,5,6,6,6\}$

Figure 14 illustrates the NURBS curve, collocation points, control points and element edges for the boundary of the dam defining the IGABEM discretisation. With IGABEM, we use two elements per line in the present case to arrive at the

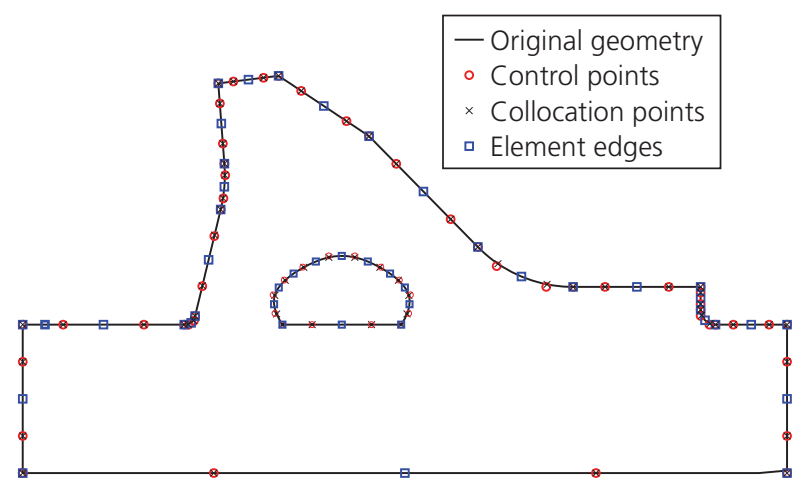

Figure 14. NURBS curve, control points, collocation points and element edges for dam problem deformed profile shown in Figure 15, where the result is compared to a FEM implementation with linear triangular elements. Once again, the IGABEM result agrees very well with the FEM.

\subsection{Propeller}

The third example applies the present method to a $3 \mathrm{D}$ propeller (Figure 16) to illustrate the ability of IGABEM to handle complex geometries. A traction of $100 \mathrm{MPa}$ is applied in the positive $z$ direction on each of the blades with zero displacement prescribed on the inner radius. Young's modulus $E$ is $100 \mathrm{GPa}$ and Poisson's ratio $v$ is $0 \cdot 3$. The initial and deformed shapes are shown in Figure 16 and the von Mises stress is illustrated in Figures 17 and 18.

This example illustrates perhaps the most important concept of this paper, which is that analysis can be performed directly on a CAD geometry without meshing, representing a significant step forward in conceptual design for engineering analysis.

\section{Conclusion}

The formulation of an isogeometric BEM for elastostatic analysis has been outlined, with the changes required over a conventional BEM implementation clearly demonstrated. The method circum-

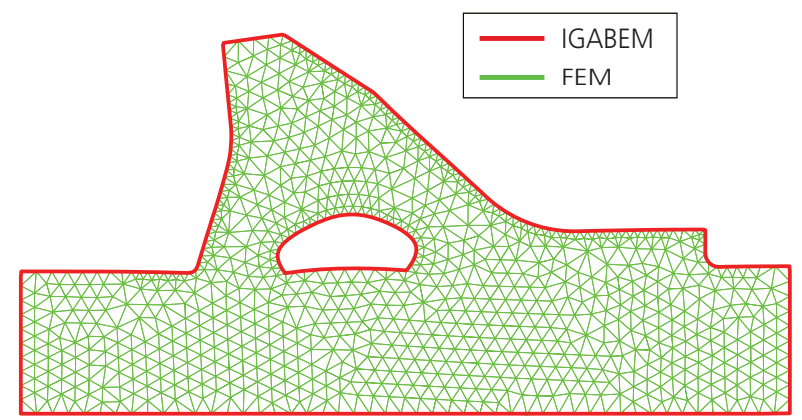

Figure 15. IGABEM and FEM dam problem: deformed shape

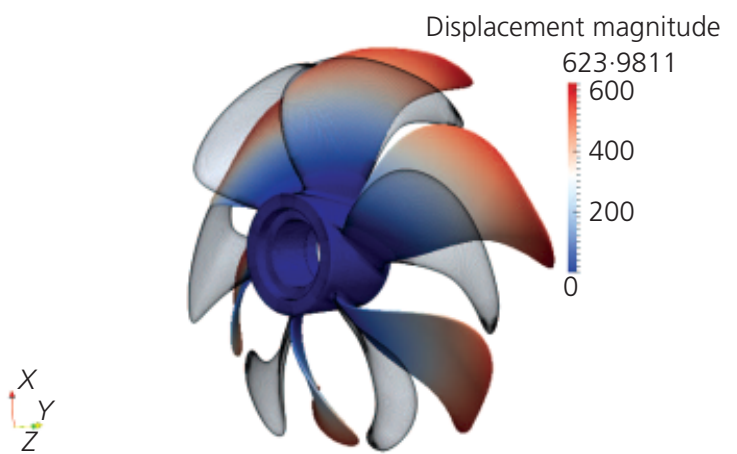

Figure 16. Propeller: geometry and deformed shape (unit: mm) 
Stress analysis without meshing: isogeometric boundary-element method Lian, Simpson and Bordas

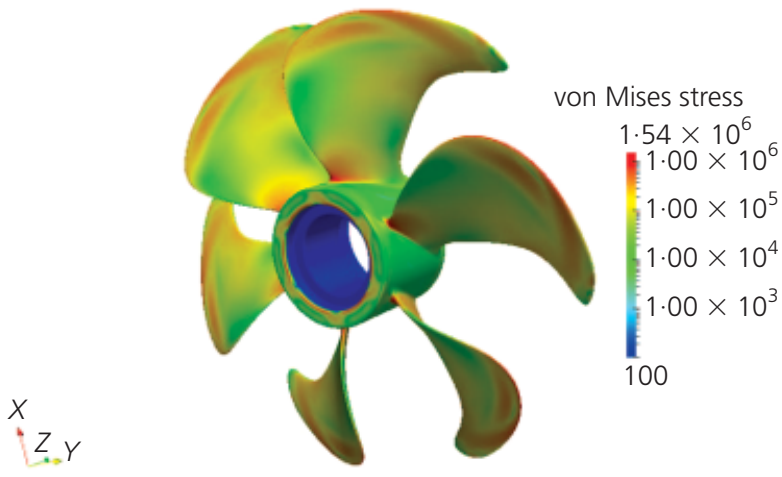

Figure 17. Propeller: von Mises stress (unit: MPa)

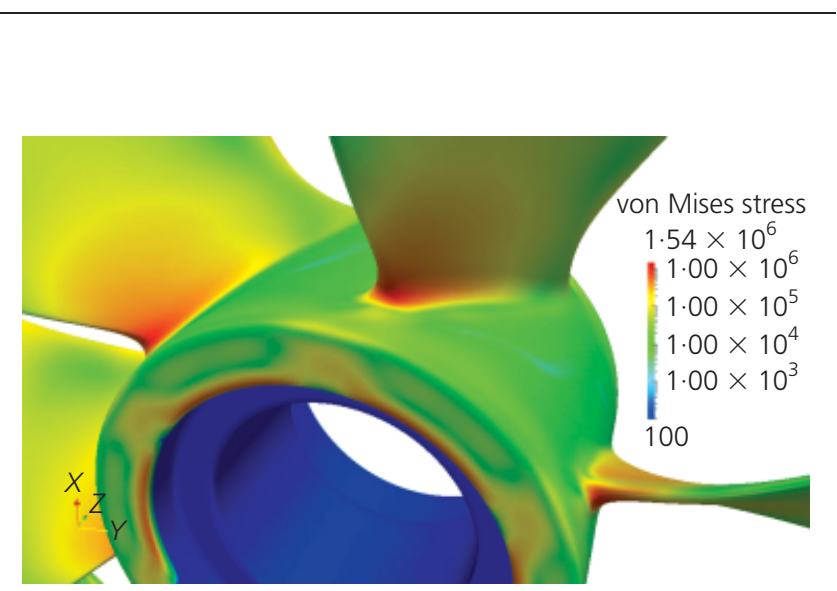

Figure 18. Close up of propeller peak von Mises stress (unit: MPa)

vents the task of mesh generation, thereby bringing the fields of design and analysis closer with significant advantages realised for early stage design. To demonstrate the accuracy of the method, examples were shown that compare against a standard boundaryelement formulation using Lagrangian basis functions and also with a standard finite-element implementation. In addition, a 3D example was presented to illustrate the ability of the method to handle extremely complex geometries without the need to generate a mesh.

\section{Appendix 1: Control points and weights of the pressure vessel}

\begin{tabular}{|c|c|c|c|}
\hline Index & $x$ & $y$ & Weight \\
\hline 1 & 0 & 0 & 1 \\
\hline 2 & 10 & 0 & 1 \\
\hline 3 & 30 & $x$ & 1 \\
\hline 4 & 40 & 0 & 1 \\
\hline 5 & 40 & $24 \cdot 8528137424$ & 0.8535533906 \\
\hline 6 & $75 \cdot 1471862576$ & 60 & 0.8535533906 \\
\hline 7 & 100 & 60 & 1 \\
\hline 8 & 100 & 70 & 1 \\
\hline 9 & 100 & 90 & 1 \\
\hline 10 & 100 & 100 & 1 \\
\hline 11 & $86 \cdot 25$ & 100 & 1 \\
\hline 12 & $58 \cdot 75$ & 100 & 1 \\
\hline 13 & 45 & 100 & 1 \\
\hline 14 & 45 & $93 \cdot 75$ & 1 \\
\hline 15 & 45 & $81 \cdot 25$ & 1 \\
\hline 16 & 45 & 75 & 1 \\
\hline 17 & 40 & 75 & 1 \\
\hline 18 & 30 & 75 & 1 \\
\hline 19 & 25 & 75 & 1 \\
\hline 20 & 25 & $66 \cdot 25$ & 1 \\
\hline 21 & 25 & $48 \cdot 75$ & 1 \\
\hline 22 & 25 & 40 & 1 \\
\hline 23 & $21 \cdot 25$ & 40 & 1 \\
\hline 24 & $13 \cdot 75$ & 40 & 1 \\
\hline 25 & 10 & 40 & 1 \\
\hline 26 & 10 & 33.75 & 1 \\
\hline 27 & 10 & $21 \cdot 25$ & 1 \\
\hline 28 & 10 & 15 & 1 \\
\hline 29 & $7 \cdot 5$ & 15 & 1 \\
\hline 30 & $2 \cdot 5$ & 15 & 1 \\
\hline 31 & 0 & 15 & 1 \\
\hline 32 & 0 & $11 \cdot 25$ & 1 \\
\hline 33 & 0 & 3.75 & 1 \\
\hline 34 & 0 & 0 & 1 \\
\hline
\end{tabular}




\section{Appendix 2: Control points and weights of the dam outer boundary}

\begin{tabular}{|c|c|c|c|}
\hline Index & $x$ & $y$ & Weight \\
\hline 1 & -3 & -20 & 1 \\
\hline 2 & $22 \cdot 75006225$ & $-20 \cdot 00000075$ & 1 \\
\hline 3 & $74 \cdot 25018675$ & $-20 \cdot 00000225$ & 1 \\
\hline 4 & $100 \cdot 000249$ & $-20 \cdot 000003$ & 1 \\
\hline 5 & $100 \cdot 0001485$ & $-15 \cdot 00000225$ & 1 \\
\hline 6 & 99.9999475 & $-5 \cdot 00000075$ & 1 \\
\hline 7 & 99.999847 & 0 & 1 \\
\hline 8 & 97.589728 & 0 & 1 \\
\hline 9 & $92 \cdot 76949$ & 0 & 1 \\
\hline 10 & $90 \cdot 359371$ & 0 & 1 \\
\hline 11 & 89.5309455321 & 0 & 0.8535533906 \\
\hline 12 & 88.359375 & $1 \cdot 1715740468$ & 0.8535533906 \\
\hline 13 & 88.359375 & $2 \cdot 000002$ & 1 \\
\hline 14 & 88.359375 & 2.76953275 & 1 \\
\hline 15 & 88.359375 & $4 \cdot 30859425$ & 1 \\
\hline 16 & 88.359375 & $5 \cdot 078125$ & 1 \\
\hline 17 & 84.06283075 & 5.078125 & 1 \\
\hline 18 & $75 \cdot 46974225$ & 5.078125 & 1 \\
\hline 19 & $71 \cdot 173198$ & $5 \cdot 078125$ & 1 \\
\hline 20 & $67 \cdot 5507928424$ & 5.078125 & 0.96105 \\
\hline 21 & $60 \cdot 8696555446$ & $7 \cdot 8807965572$ & 0.96105 \\
\hline 22 & $58 \cdot 331384$ & $10 \cdot 46514$ & 1 \\
\hline 23 & $54 \cdot 66650675$ & $14 \cdot 19651125$ & 1 \\
\hline 24 & $47 \cdot 33675225$ & 21.65925375 & 1 \\
\hline 25 & 43.671875 & $25 \cdot 390625$ & 1 \\
\hline 26 & 40.625 & $27 \cdot 421875$ & 1 \\
\hline 27 & 34.53125 & 31.484375 & 1 \\
\hline 28 & $31 \cdot 484375$ & $33 \cdot 515625$ & 1 \\
\hline 29 & $29 \cdot 453125$ & $33 \cdot 26171875$ & 1 \\
\hline 30 & $25 \cdot 390625$ & $32 \cdot 75390625$ & 1 \\
\hline 31 & 23.359375 & $32 \cdot 5$ & 1 \\
\hline 32 & 23.563557 & 29.7959275 & 1 \\
\hline 33 & 23.971921 & 24.3877825 & 1 \\
\hline 34 & $24 \cdot 176103$ & $21 \cdot 68371$ & 1 \\
\hline 35 & 24.2935527074 & $20 \cdot 1282857015$ & 0.993954 \\
\hline 36 & 24.0432981685 & $17 \cdot 0186352549$ & 0.993954 \\
\hline 37 & $23 \cdot 67862$ & $15 \cdot 502011$ & 1 \\
\hline 38 & $22 \cdot 815829$ & 11.91383875 & 1 \\
\hline 39 & $21 \cdot 1939395728$ & $5 \cdot 1687305659$ & 0.8927115 \\
\hline 40 & $20 \cdot 227456$ & $1 \cdot 149322$ & 0.785423 \\
\hline 41 & $20 \cdot 0726719675$ & 0.505591894 & 0.8927115 \\
\hline 42 & $19 \cdot 3600570142$ & $-4.26852132573430 \times 10^{-6}$ & 1 \\
\hline 43 & $18 \cdot 769013$ & 0 & 1 \\
\hline 44 & $13 \cdot 32675975$ & 0 & 1 \\
\hline 45 & $2 \cdot 44225325$ & 0 & 1 \\
\hline 46 & -3 & 0 & 1 \\
\hline 47 & -3 & -5 & 1 \\
\hline 48 & -3 & -15 & 1 \\
\hline 49 & -3 & -20 & 1 \\
\hline
\end{tabular}




\section{Appendix 3: Control points and weights of the dam inner boundary}

\begin{tabular}{llll}
\hline Index & $x$ & $y$ & Weight \\
\hline 1 & 32 & 0 & 1 \\
2 & 31 & $1 \cdot 5$ & 1 \\
3 & $30 \cdot 75$ & 4 & 1 \\
4 & $32 \cdot 25$ & 6 & 1 \\
5 & $34 \cdot 75$ & $7 \cdot 75$ & 1 \\
6 & $38 \cdot 25$ & $9 \cdot 25$ & 1 \\
7 & $41 \cdot 75$ & $9 \cdot 25$ & 1 \\
8 & $45 \cdot 25$ & $7 \cdot 75$ & 1 \\
9 & $47 \cdot 75$ & 6 & 1 \\
10 & $49 \cdot 25$ & 4 & 1 \\
11 & 49 & $1 \cdot 5$ & 1 \\
12 & 48 & 0 & 1 \\
13 & 44 & 0 & 1 \\
14 & 36 & 0 & 1 \\
15 & 32 & 0 & 1 \\
\hline
\end{tabular}

\section{REFERENCES}

Banerjee PK and Butterfield R (1981) Boundary Element Methods in Engineering Science. McGraw-Hill, New York, NY, USA.

Benson DJ, Bazilevs Y, Hsu MC and Hughes TJR (2010) Isogeometric shell analysis: the Reissner-Mindlin shell. Computer Methods in Applied Mechanics and Engineering 199(5/8): 276-289.

Buffa A, Sangalli G and Vázquez R (2010) Isogeometric analysis in electromagnetics: B-splines approximation. Computer
Methods in Applied Mechanics and Engineering 199(17/20): $1143-1152$.

Cottrell JA, Reali A, Bazilevs Y and Hughes TJR (2006) Isogeometric analysis of structural vibrations. Computer Methods in Applied Mechanics and Engineering 195(41/43): 5257-5296.

Cox MG (1971) The numerical evaluation of $B$-splines. IMA Journal of Applied Mathematics 10(2): 134-179.

de Boor C (1972) On calculating with B-splines. Journal of Approximation Theory 6(1): 50-62.

Hughes TJR, Cottrell JA and Bazilevs Y (2005) Isogeometric analysis: CAD, finite elements, NURBS, exact geometry and mesh refinement. Computer Methods in Applied Mechanics and Engineering 194(39/41): 4135-4195.

PiegI LA and Tiller W (1997) The NURBS Book. Springer, Berlin, Germany.

Sederberg T, Zheng J, Bakenov A and Nasri A (2003) T-splines and T-NURCCs. ACM Transactions on Graphics 22(3): $477-$ 484.

Simpson RN, Bordas SPA, Trevelyan J and Rabczuk T (2012) A two-dimensional isogeometric boundary element method for elastostatic analysis. Computer Methods in Applied Mechanics and Engineering 209-212: 87-100.

Strang G and Fix G (1973) An Analysis of the Finite Element Method. Prentice-Hall, Englewood Cliffs, NJ, USA.

Temizer I, Wriggers P and Hughes TJR (2010) Contact treatment in isogeometric analysis with NURBS. Computer Methods in Applied Mechanics and Engineering 200(9/12): 1100-1112.

Wall WA, Frenzel MA and Cyron C (2008) Isogeometric structural shape optimization. Computer Methods in Applied Mechanics and Engineering 197(33/40): 2976-2988.

Zienkiewicz OC (1971) The Finite Element Method in Engineering Science. McGraw-Hill, London, UK.
WHAT DO YOU THINK?

To discuss this paper, please email up to 500 words to the editor at journals@ice.org.uk. Your contribution will be forwarded to the author(s) for a reply and, if considered appropriate by the editorial panel, will be published as a discussion in a future issue of the journal.

Proceedings journals rely entirely on contributions sent in by civil engineering professionals, academics and students. Papers should be 2000-5000 words long (briefing papers should be 1000-2000 words long), with adequate illustrations and references. You can submit your paper online via www.icevirtuallibrary.com/content/journals, where you will also find detailed author guidelines. 